\title{
Degarelix treatment is compatible with diabetes and antithrombotic therapy in patients with prostate cancer
}

This article was published in the following Dove Press journal: Research and Reports in Urology

\author{
Suguru Tokiwa' \\ Hiroaki Shimmura' \\ Shuhei Nomura ${ }^{2-4}$ \\ Ryota Watanabe' \\ Minoru Kurita' \\ Naoto Yoshida' \\ Kaori Yamashita' \\ Yoshitaka Nishikawa ${ }^{5}$ \\ Alexander Kouzmenko 6 \\ Shigeaki Kato ${ }^{4}$ \\ 'Department of Urology, Jyoban \\ Hospital, Tokiwa Foundation, Iwaki, \\ Fukushima, Japan; ${ }^{2}$ Department of \\ Epidemiology and Biostatistics, School \\ of Public Health, Imperial College \\ London, London, UK; ${ }^{3}$ Department \\ of Global Health Policy, Graduate \\ School of Medicine, The University \\ of Tokyo, Tokyo, ${ }^{4}$ Research Institute \\ of Innovative Medicine, Tokiwa \\ Foundation, Iwaki, ${ }^{5}$ Department of \\ Health Informatics, School of Public \\ Health, Kyoto University, Kyoto, Japan; \\ ${ }^{6}$ Department of Life Sciences, Alfaisal \\ University, Riyadh, Kingdom of Saudi \\ Arabia
}

Correspondence: Suguru Tokiwa Department of Urology, Jyoban Hospital, Tokiwa Foundation, 57 Kaminodai, Jyoban Kamiyunagayamachi, Iwaki 972-8322, Fukushima, Japan

Tel +8I 246434175

Email suguru-tokiwa@tokiwa.or.jp

Shigeaki Kato

Research Institute of Innovative Medicine, Jyoban Hospital, Tokiwa Foundation, 57 Kaminodai, Jyoban Kamiyunagayamachi, Iwaki 972-8322, Fukushima, Japan

Email uskato0525@gmail.com
Introduction: Therapeutically induced androgen deficiency (AD) is a standard treatment for patients with prostate cancer, but it is often associated with various adverse effects (AEs) that may lead to discontinuation. Some AEs may depend on the patient's health condition, while others may be due to complications of the drug delivery method. Degarelix is a gonadotropinreleasing hormone $(\mathrm{GnRH})$ antagonist widely used for the treatment of androgen-dependent prostate cancer. This study aimed to ascertain the following: 1) the compatibility of degarelix treatment with diabetes and 2) any specific causal associations of degarelix injections with increased blood clotting and antithrombotic therapy requirements.

Patients and methods: The medical records of 162 patients with prostate cancer who had undergone degarelix treatment were retrospectively examined. The association of a medical history of diabetes and anticoagulant co-treatment with degarelix treatment discontinuation was analyzed statistically.

Results: Rapid and significant decreases in prostate-specific antigen (PSA) levels during the course of degarelix treatment were detected for patients with prostate cancer regardless of clinical state. During the 27 months of treatment, 68 subjects $(48 \%)$ ceased degarelix treatment, owing to several reasons, mainly financial issues. Among these subjects, 19 had diabetes, while 35 were treated with antithrombotics. Extensive statistical analysis indicated that there were no causal associations between degarelix treatment discontinuation and preexisting diabetes or antithrombotic therapy.

Conclusion: Our study suggests that preexisting diabetes and antithrombotic therapy were not significant factors for the discontinuation of degarelix treatment in patients with prostate cancer. Keywords: GnRH antagonists, prostate cancer, degarelix, discontinuation, diabetes, antithrombotic treatment

\section{Introduction}

Induction of androgen deficiency (AD) is the most commonly used treatment for patients with advanced/metastatic prostate cancer, since the development of prostate cancer is dependent on activated androgen signaling. ${ }^{1,2} \mathrm{An} \mathrm{AD}$ state is achievable surgically (by bilateral orchidectomy) or therapeutically (by blunting androgen production via gonadotropin-releasing hormone [ $\mathrm{GnRH}]$-facilitated luteinizing hormone $[\mathrm{LH}]$ and follicle-stimulating hormone [FSH; ie, GnRH-LH/FSH axis]) to debilitate androgen receptor function. ${ }^{1-3}$ Furthermore, the function of androgen receptors can be inhibited by androgen antagonists. ${ }^{4,5}$ Since GnRH is the most upstream endocrine inducer of androgen production, GnRH agonists were first developed for AD therapy to establish a negative feedback regulation of androgen production. ${ }^{6,7}$ However, the potential for non-physio- 
logical rapid increases in testosterone levels in patients treated with GnRH agonists, leading to androgen receptor activation in prostate tumors and further tumor growth stimulation, is a significant concern. ${ }^{8,9} \mathrm{GnRH}$ antagonists thus emerged to achieve the $\mathrm{AD}$ state without detrimental testosterone surges and consequent increases in serum prostate-specific antigen (PSA) levels in treated patients. ${ }^{10-12}$ Currently, one GnRH antagonist, degarelix, is widely used for AD induction therapy and has been proven to be at least as efficient as some known GnRH and LH agonists by many indicators of clinical efficacy, but without their undesirable side effects. ${ }^{13,14}$

Similar to other hormone agonists and antagonists indicated for AD therapy, degarelix is also known to cause complications, mainly related to AD. ${ }^{13,14}$ Therapeutically induced AD is commonly associated with typical adverse effects (AEs) such as hot flushes, fatigue and weight gain, regardless of the specific therapeutic compound administered. ${ }^{1,2}$ As the physiological actions of androgens facilitate basal metabolism in males via energy expenditure more efficiently than in females, ${ }^{15,16}$ AD therapy has been suggested to be risky in patients with diabetes. Thus, AD therapy has been believed to possibly lead to undesired health complications in treated prostate cancer patients. ${ }^{1-3}$ Particularly, the occurrence of severe AEs often deters patients from continuing therapy, leading to discontinuation. Specific AEs have also been associated with degarelix, presumably related to the route of administration. ${ }^{17-20}$ As degarelix is given by repeated injections, injection site reactions, such as pain, erythema, swelling and nodules, have been noted as AEs. Since severe injection site skin reactions and related complications may force patients to discontinue ongoing therapy, ${ }^{18,19}$ it is beneficial for the patients with prostate cancer to minimize any baseline risk that may contribute to the occurrence of undesired AEs, including injection site reactions.

Skin is prone to hypersensitivity in patients with certain conditions and/or drug treatments for diseases other than prostate cancer. Patients with diabetes are highly susceptible to skin complications, such as bacterial/fungal infections, severe irritation and itching. ${ }^{21,22}$ Such clinical preconditions could be serious confounding factors for treatments requiring repeated injections. Antithrombotic treatment is also often used for elderly patients, including prostate cancer patients, and may lead to eventual bleeding. ${ }^{23,24}$ As serious AEs, including skin complications induced by repeated degarelix injections, lead to discontinuation of therapy, ${ }^{18,19}$ the current retrospective study was undertaken to assess whether diabetes and/or antithrombotic treatment were risk factors for discontinuation of degarelix therapy in patients with prostate cancer with a Gleason score higher than 6 .

\section{Patients and methods Data sources}

We retrospectively examined the records of 162 patients with prostate cancer treated with degarelix at the Jyoban Hospital (Iwaki, Japan) from November 2012 to October 2015. The subjects were classified into four subgroups, three of which depended on Gleason scores and serum PSA levels (ie, D'Amico's method that stratifies patients with localized prostate cancer into low, intermediate or high risk of biochemical recurrence $)^{25}$ and one subgroup of patients with advanced prostate cancer with metastasis to other organs. The patients with advanced prostate cancer bearing metastatic tumors were also treated with other drugs, such as docetaxel, enzalutamide, or abiraterone, to inhibit further cancer cell proliferation.

Degarelix was periodically injected subcutaneously according to the recommended protocol: $240 \mathrm{mg}$ for the first injection and then $80 \mathrm{mg}$ every month. We assessed baseline characteristics, ongoing and past therapy, and switches to other drugs and therapy. To evaluate the treatment response, we monitored the serum PSA levels $(\mathrm{ng} / \mathrm{mL}$ ).

Diabetes mellitus was diagnosed according to the criteria of the Japanese Diabetes Society. ${ }^{26}$

Antithrombotic therapy included the following drugs: antiplatelet agents (eg, aspirin, clopidogrel, cilostazol, ticlopidine, eicosapentaenoic acid, and limaprost alfadex) and anticoagulants with vitamin $\mathrm{K}$ inhibition (eg, warfarin) or direct factor Xa inhibition (eg, rivaroxaban). ${ }^{27}$

\section{Statistical analysis}

To evaluate the crude relationship between having diabetes and receiving an antithrombotic treatment and discontinuation of degarelix therapy, we considered the Kaplan-Meier product limit method. ${ }^{26,27}$ The Kaplan-Meier method is a widely used retrospective technique to estimate the cumulative probability of event occurrence (ie, discontinuation of degarelix therapy) at a particular follow-up time, which is also known as a probability curve. Using the Wilcoxon test and the log-rank test, ${ }^{28,29}$ we compared the estimated probability curves between subjects with or without diabetes and between those who received or did not receive antithrombotic treatment.

A Cox proportional hazards regression model - one of the most common methods used in time-to-event data analysis ${ }^{28,29}$ - was also employed to evaluate the relationship between having diabetes and receiving an antithrombotic treatment and discontinuation of degarelix therapy, with adjustment for covariates. Independent variables considered in the model were prostate cancer subgroups (low, interme- 
diate or high risk of recurrence in localized prostate cancer according to D'Amico risk stratification, or metastatic cancer), age at diagnosis, initial pretreatment PSA level (ng/ $\mathrm{mL}$ ) and experience of radical prostatectomy.

All statistical analyses were conducted using STATA/ IC 14 (StataCorp LLC, College Station, TX, USA), and a $p$-value of less than 0.05 was considered statistically significant. The datasets are available on reasonable request to the corresponding authors.

\section{Ethics}

This study underwent ethical review and was approved by the Jyoban Hospital Institutional Review Board. All study participants provided written informed consent.

\section{Results}

\section{Patient characteristics}

The baseline characteristics of the studied patients are summarized in Table 1. Among the 162 subjects, 114 subjects with localized prostate cancer were classified into subgroups of low $(n=14)$, intermediate $(n=31)$ and high risk $(n=69)$ of recurrence, based on Gleason scores and serum PSA levels, and the remaining subjects $(n=48)$ had metastatic tumors. The mean age and SD of the four subgroups at diagnosis were 72.1 (5.2), 74.6 (7.2), 76.6 (6.3) and 74.2 (9.5) years, respectively, which were not statistically significantly different ( $p=0.66$ : ANOVA). The minimum age was 48 years, and the maximum age was 89 years. The mean (standard deviation: SD) initial PSA levels were 6.2 (1.9), 10.4 (4.7), 31.8 (36.5) and 390.9 (360.3) ng/mL, respectively, which were statistically significantly different $(p<0.001$ : ANOVA). Note that nine subjects with metastasis ( $18.8 \%$ of 48 ) had initial PSA levels >999 ng/mL, which were scored as "999" by default.
This means that the mean initial PSA level for the metastasis group was underestimated. The Gleason scores for low- and intermediate-risk groups were within 7 , but they were more than 8 for the high-risk and metastasis groups. Two cases in the high-risk group and six cases in the metastasis group had undergone prior therapies before degarelix administration.

The PSA response to degarelix therapy is shown in Figure 1. In the three subgroups of patients with localized prostate cancer, the PSA levels decreased sharply in the first 3 months, and gradual declines continued over 1 year. Up to the assessed 27 months, the PSA levels were maintained at normal levels (about $4 \mathrm{ng} / \mathrm{mL}$ ) for these three subgroups. Degarelix therapy was also effective for the group with metastasis, as a similar sharp drop in PSA levels was also detectable in the first 3 months, but they remained high (more than 4 $\mathrm{ng} / \mathrm{mL}$ ), even when assessed after 27 months of treatment.

\section{Discontinuation of degarelix use}

Nearly half of all subjects $(n=68: 42.0 \%)$ ceased degarelix use. The reasons for discontinuation of degarelix therapy are summarized in Table 2. The most common causes of discontinuation were not serious AEs, but switches to the other drugs ( $n=12: 7.4 \%)$, or due to drugs not being effective $(n=10$ : $6.2 \%)$. Nine patients $(5.6 \%)$ discontinued degarelix because of AEs, including five (3.1\%) severe injection site reactions.

\section{Probability of discontinuation of degarelix use}

We then assessed if an association existed between other clinical conditions and degarelix therapy discontinuation. Although injection site reaction AEs were not raised as major reasons for discontinuation, diabetes and antithrombotic treatment might have exerted an influence on enhancing

Table I Patient characteristics $(n=162)$

\begin{tabular}{|c|c|c|c|c|}
\hline \multirow[t]{2}{*}{ Characteristics } & \multicolumn{4}{|l|}{ Group } \\
\hline & Low risk & Intermediate risk & High risk & Metastasis \\
\hline Number of cases & 14 & 31 & 69 & 48 \\
\hline Age (years), mean (SD) & $72.1(5.2)$ & $74.6(7.2)$ & $76.6(6.3)$ & $74.2(9.5)$ \\
\hline Initial PSA (ng/mL), mean (SD) & $6.2(1.9)$ & $10.4(4.7)$ & $31.8(36.5)$ & $390.9(360.3)$ \\
\hline \multicolumn{5}{|l|}{ Gleason score } \\
\hline 6 & 14 & I & 2 & - \\
\hline 7 & - & 30 & 14 & I \\
\hline More than 8 & - & - & 54 & 42 \\
\hline Previous treatment & - & - & 2 & 6 \\
\hline \multicolumn{5}{|l|}{ Combination of drug } \\
\hline Docetaxel & - & - & - & 4 \\
\hline Enzalutamide & - & - & I & 9 \\
\hline Abiraterone & - & - & - & 4 \\
\hline
\end{tabular}

Abbreviation: PSA, prostate-specific antigen. 
A

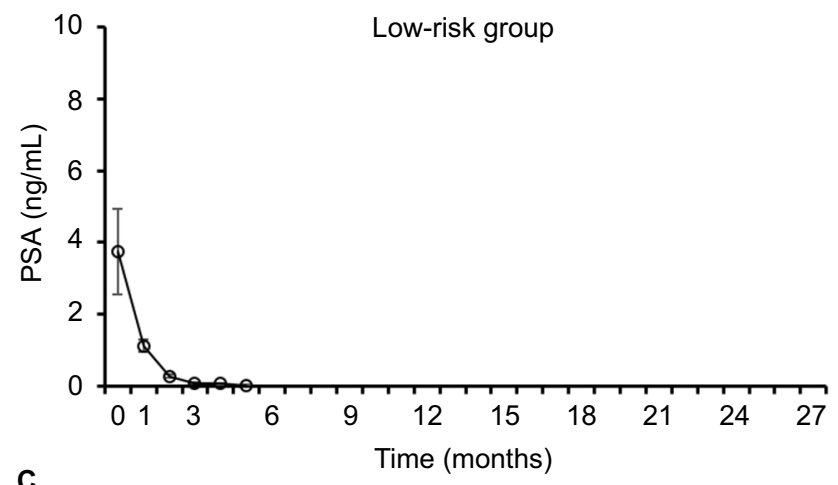

C

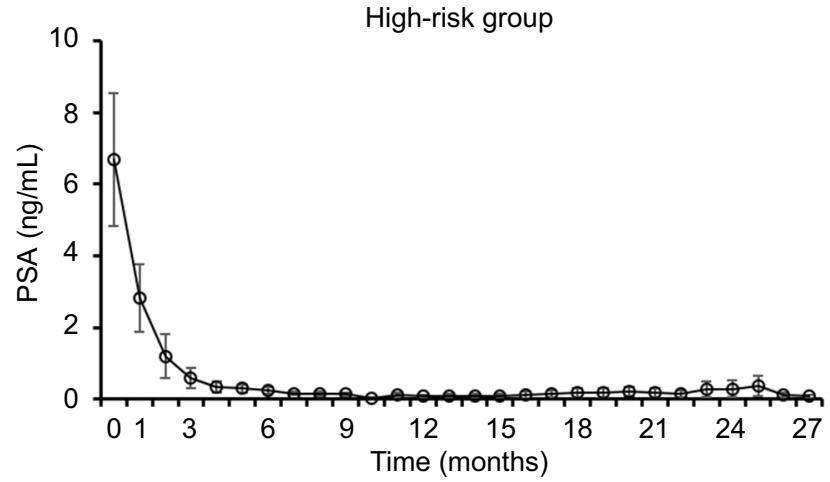

B

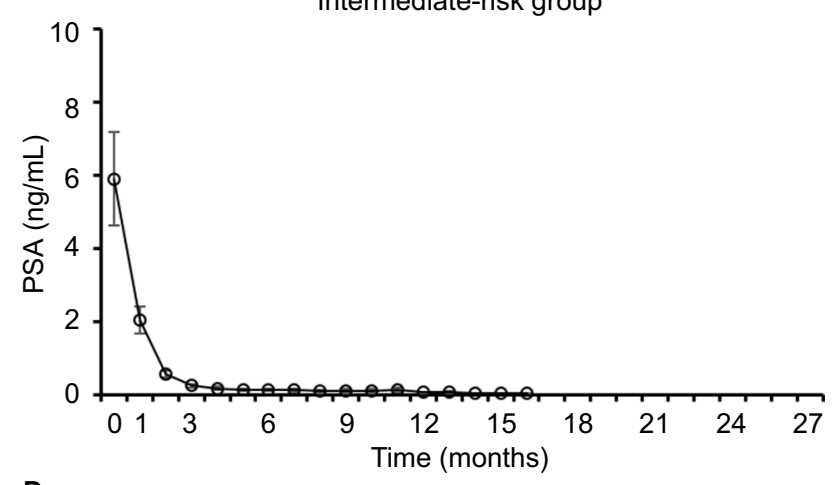

D

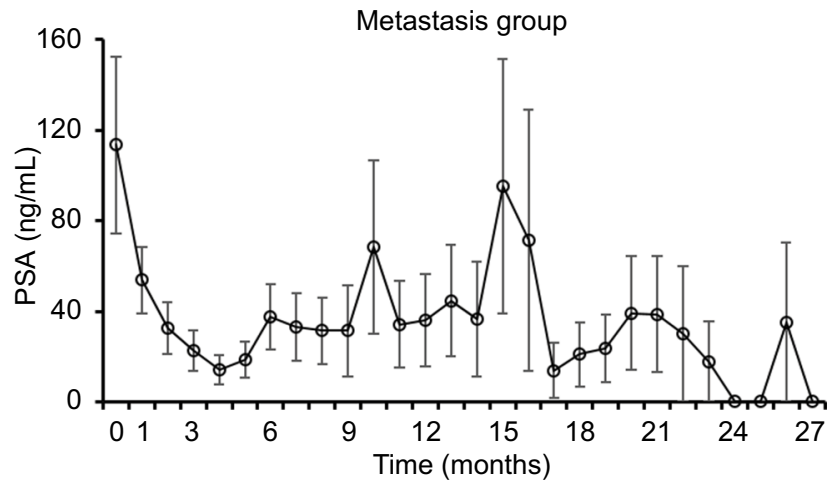

Figure I PSA response to degarelix therapy in the (A) low-risk group, (B) intermediate-risk group, (C) high-risk group and (D) metastasis group. Abbreviation: PSA, prostate-specific antigen.

Table 2 Summary of reasons for discontinuation of degarelix therapy

\begin{tabular}{|c|c|c|c|c|}
\hline \multirow[t]{2}{*}{ Reasons } & \multicolumn{4}{|l|}{ Group } \\
\hline & Low risk & Intermediate risk & High risk & Metastasis \\
\hline Discontinuation cases/total cases & $4 / 14$ & $9 / 31$ & $36 / 69$ & $19 / 48$ \\
\hline \multicolumn{5}{|l|}{ Adverse events } \\
\hline Injection site reaction & - & I & 3 & I \\
\hline Hot flash & - & I & - & - \\
\hline Malaise & - & I & - & I \\
\hline Suspicion of hepatic disorder & - & - & I & - \\
\hline Change to other drugs & - & I & 11 & - \\
\hline Drugs ineffective & - & - & 2 & 8 \\
\hline Deaths & - & - & 2 & 1 \\
\hline Difficulty in attending & 2 & 1 & 1 & - \\
\hline Others* & 2 & 4 & 16 & 8 \\
\hline Period until discontinuation (day), mean (SD) & II 6.3 (I39.7) & $268.7(176.1)$ & $349.9(204.8)$ & $370.5(300.0)$ \\
\hline
\end{tabular}

Note: *Others include combination with high-intensity focused ultrasound or radiotherapy.

serious AEs that eventually led to discontinuation of degarelix therapy. Figure 2 shows the Kaplan-Meier curves of the probability of discontinuation of degarelix therapy. The probability was plotted separately in subjects with or without diabetes (the upper graph in Figure 2A) and in those who received or did not receive an antithrombotic treatment (the lower graph in Figure 2B), against the analysis time that started from the date of the initial administration of degarelix.

No significant difference in probability curves for discontinuation of degarelix therapy was observed between subjects with or without diabetes and those who received or did not receive an antithrombotic treatment. The $p$-values from the Wilcoxon test were 0.54 and 0.35 , respectively, and from the log-rank test wre 0.44 and 0.23 , respectively.

\section{Regression model for discontinuation of degarelix use}

The results of the multiple Cox proportional hazards regression model are summarized in Table 3 and are expressed as a multiplicative change (ie, hazard ratio) in the likelihood 
A

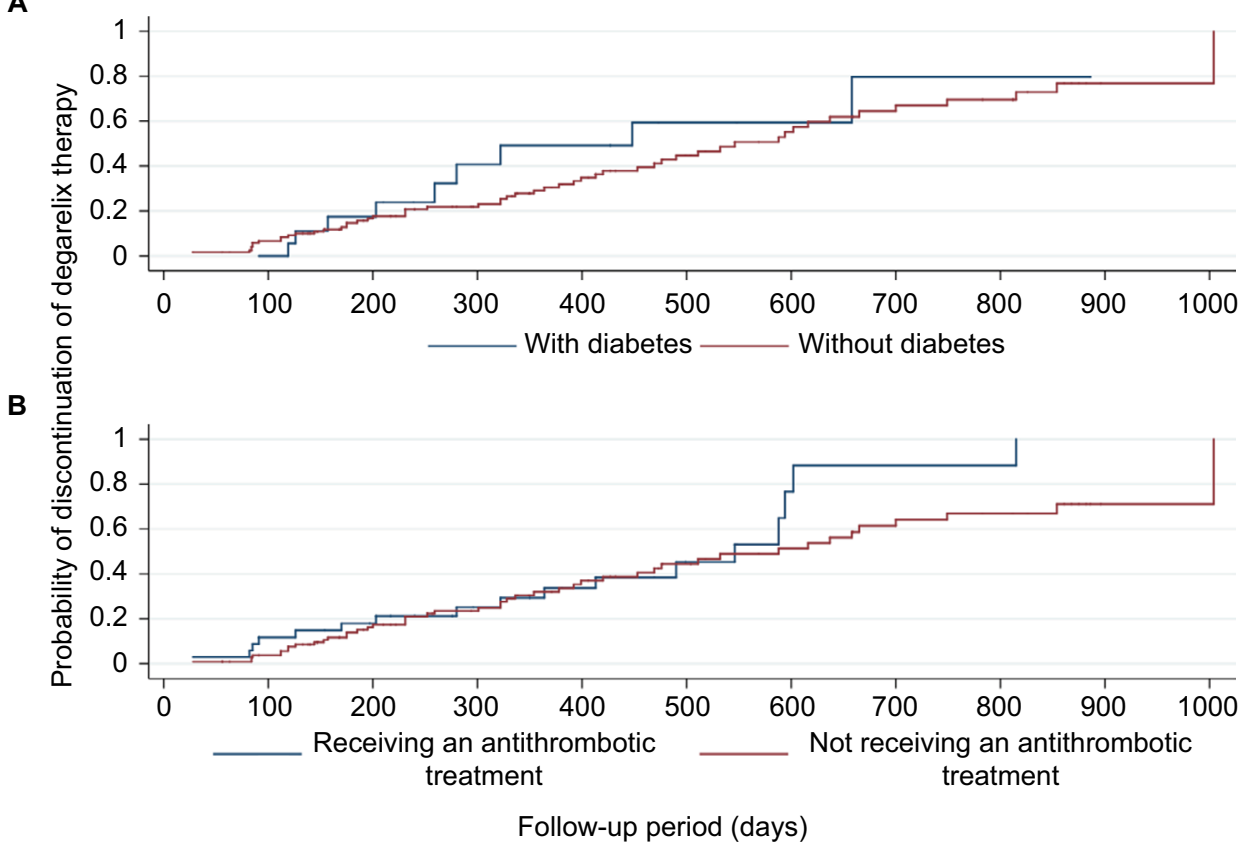

Figure 2 Estimated probability of discontinuation of degarelix therapy by diabetes status $(\mathbf{A})$ and anticoagulant treatment conditions (B).

Table 3 Multiple Cox regression model showing the effect of being diabetic and receiving an antithrombotic treatment on discontinuation of degarelix therapy

\begin{tabular}{llll}
\hline Variable & $\begin{array}{l}\text { Hazard } \\
\text { ratio }\end{array}$ & $95 \%$ Cl & $p$-value \\
\hline Diabetes status & & & \\
$\quad$ No & 1.00 & & \\
$\quad$ Yes & 1.19 & $0.51-2.75$ & 0.69 \\
Antithrombotic treatment & & & \\
$\quad$ No & 1.00 & & \\
$\quad$ Yes & 1.63 & $0.89-2.98$ & 0.11 \\
Cancer risk group & & & \\
$\quad$ Low risk & 0.79 & $0.14-4.33$ & 0.78 \\
$\quad$ Intermediate risk & 1.33 & $0.48-3.76$ & 0.58 \\
$\quad$ High risk & 1.42 & $0.62-3.24$ & 0.41 \\
$\quad$ Metastasis & 1.00 & & \\
Age at diagnosis & 0.99 & $0.95-1.03$ & 0.49 \\
Initial PSA levels (per I ng/mL) & 1.00 & $1.00-1.00$ & 0.46 \\
Radical prostatectomy surgery & & & \\
$\quad$ Not experienced & 1.00 & & \\
$\quad$ Experienced & 4.69 & $2.32-9.49$ & $<0.001$ \\
\hline
\end{tabular}

Abbreviation: PSA, prostate-specific antigen.

of discontinuation of degarelix therapy for a unit increase/ change in the independent variable.

The model that we specified is the following:

$$
h(t)=h_{0}(t) \exp \left(\begin{array}{l}
\beta_{1} \times X_{1}+\beta_{2} \times X_{2}+\beta_{3} \times X_{3} \\
+\beta_{4} \times X_{4}+\beta_{5} \times X_{5}+\beta_{6} \times X_{6}
\end{array}\right)
$$

where $h(t)$ is the hazard function, $h_{0}(t)$ is the baseline hazard function at time $t$ and $\exp (\beta)$ indicates the hazard ratio. The model incorporated six covariates $\left(\mathrm{X}_{1}\right.$ : diabetes status - no or yes; $\mathrm{X}_{2}$ : antithrombotic treatment conditions - no or yes; $\mathrm{X}_{3}$ : cancer risk levels - low, intermediate or high risk of recurrence for localized cancer, or metastatic cancer; $\mathrm{X}_{4}$ : age at diagnosis; $\mathrm{X}_{5}$ : initial PSA levels and $\mathrm{X}_{6}$ : radical prostatectomy - not experienced or experienced). Our major interests were comparisons between "no" and "yes" with respect to the covariates "diabetes status" and "antithrombotic treatment," effects that were expressed as $\beta_{1}$ and $\beta_{2}$, respectively. This model takes into account the time from the date of the initial administration of degarelix until its discontinuation or the last date of follow-up.

After adjusting for covariates, having diabetes and receiving an antithrombotic treatment had no significant relationship with discontinuation of degarelix therapy, with hazard ratios of $1.19(95 \%$ CI $0.51-2.75, p=0.69)$ and 1.63 (95\% CI 0.89-2.98, $p=0.11$, respectively. Radical prostatectomy was the only variable significantly associated with discontinuation of degarelix therapy (hazard ratio $4.69,95 \%$ CI $2.32-9.49, p<0.001$ ).

\section{Discussion}

GnRH antagonists were developed to overcome the complications of the GnRH agonists that caused a rapid surge of androgen production and consequent exacerbation of prostate tumors. ${ }^{13,14}$ Numerous clinical studies have confirmed that a GnRH antagonist, degarelix, successfully induced an AD state without a transient increase in serum testosterone levels, and with a robust decrease in serum PSA levels in patients with 
prostate cancer of different stages. ${ }^{10-12}$ Consistently, in the current study, the expected PSA response was seen in patients of all subgroups. Since the serum PSA level is a better clinical marker for prostate cancer therapy than serum testosterone levels, serum testosterone levels were not systemically assessed in the treated patients. However, it should be noted that monitoring serum testosterone levels in patients with prostate cancer can also be very informative to predict cancer progression, as endogenous production of androgens is dysregulated during the treatments. ${ }^{30-32}$ Previous studies suggested that repeated degarelix injections might provoke skin reactions at the injection site, particularly at the first injection. ${ }^{10-12}$ It has been estimated that injection site reactions occur in over $40 \%$ of degarelix-treated patients ${ }^{11}$ and that they eventually become serious enough to cause discontinuation of therapy. In the current study, we also observed that large numbers of patients developed injection site reactions of varying severity, but they only caused discontinuation of degarelix therapy in four cases. Thus, degarelix therapy may cause injection site reactions, but they may not be serious enough to prompt discontinuation from the perspective of both patients and doctors.

Previous reports of frequent AEs among treated patients led us to question whether these serious AEs resulted in discontinuation in our study, as the major causes had been potentiated by the preexisting conditions of the treated patients. We focused specifically on patients with documented clinical conditions of higher susceptibility to any skin stimulation, since their skin conditions would be likely to deteriorate in response to regular injections, leading to potentially serious AEs in skin and eventual treatment discontinuation. Although a high frequency of injection site reactions has been observed in many studies, ${ }^{10-12}$ information about clinical associations between the occurrence of the injection site reactions and other disease conditions is scarce. We particularly focused on common diseases and treatments that might potentiate the occurrence of AEs during degarelix therapy. In this study, we assessed whether there was an association between discontinuation of degarelix therapy and diabetes, since the skin in patients with diabetes is known to be hypersensitive to any stimulation ${ }^{21,22}$ and, therefore likely to develop serious AEs resulting in discontinuation of any nonvital treatment. Similarly, co-treatment with antithrombotic agents is also assumed to potentiate the occurrence of AEs in response to degarelix injections, since antithrombotic treatment is well known to often cause side effects related to bleeding in the skin and other organs. ${ }^{23,24}$

Nevertheless, a relationship between diabetes and the incidence of AEs related to degarelix therapy was not found. Similarly, a causal association of AEs with degarelix therapy and antithrombotic treatment remains to be ascertained. There were 19 subjects among the tested 148 subjects with prostate cancer who were clinically diabetic. We could determine no statistical association between degarelix treatment discontinuation and diabetes in this group of patients (Figure 2 and Table 3). Thus, the incidence of common AEs, including injection site reactions, is unlikely to be influenced by diabetes. However, the relationship between AEs and body mass index (BMI) still remains to be tested, due to lack of the BMI data for this cohort, as local androgen actions for cancer progression have been indicated to be potentiated by the increased adipocyte tissues.,33 Likewise, under the same statistical analyses, antithrombotic co-treatment was not causal for discontinuation of degarelix therapy (Figure 2 and Table 3). At the same time, we observed a statistically significant association of degarelix therapy discontinuation with radical prostatectomy, but the basis of this association remains to be addressed in further studies.

In this study, we analyzed the potential for a causal association between degarelix therapy discontinuation and the most common preexisting condition (diabetes) or cotreatment (antithrombotic therapy) in a cohort of prostate cancer patients. It is possible that other common diseases and co-therapies may lead to the development of different serious AEs that would eventually lead to discontinuation of degarelix therapy. To address this issue, further studies on larger cohorts of subjects are clearly required to optimize therapeutic strategies for degarelix treatment in patients with prostate cancer to avoid undesired AEs and unnecessary discontinuation of treatment.

The limitations of this study are the very small number of subjects as well as its retrospective nature. Although a recent paper reported a genetic effect by polymorphism in the gene locus of aldo-keto reductase $1 \mathrm{C} 3,{ }^{34}$ we were unable to assess whether genetic variations were associated with the AEs. The diabetic condition and antithrombotic therapy among the studied patients were diverse, but for the degarelix effect assessment, the patients were simply classified into four groups based on the prostate cancer states evaluated by D'Amico's method.

\section{Conclusion}

Degarelix effectively induced an AD state in patients with prostate cancer, and frequent occurrence of AEs was observed, in accordance with past reports. However, preexisting diabetes and antithrombotic therapy were not statistically significant predictors for the discontinuation of degarelix treatment in patients with prostate cancer. 


\section{Acknowledgment}

The authors thank all the staff members of the Jyoban Hospital who conducted clinical treatment and acknowledge Dr Toshihide Horiuchi, Dr. Hisao Kurihara, Mr Tomonori Kimura, Mr Koichi Nishimura, Ms Claire Leppold and Dr Michio Tokiwa for supporting the clinical analyses and manuscript preparation.

\section{Disclosure}

The authors report no conflicts of interest in this work.

\section{References}

1. AttardG,ParkerC,EelesRA, etal.Prostate cancer.Lancet.2016;387(10013): 70-82.

2. Li Z, Alyamani M, Li J, et al. Redirecting abiraterone metabolism to fine-tune prostate cancer anti-androgen therapy. Nature. 2016;533(7604):547-551.

3. Sharifi N. Mechanisms of androgen receptor activation in castrationresistant prostate cancer. Endocrinology. 2013;154(11):4010-4017.

4. Scher HI, Fizazi K, Saad F, et al; AFFIRM Investigators. Increased survival with enzalutamide in prostate cancer after chemotherapy. N Engl J Med. 2012;367(13):1187-1197.

5. de Bono JS, Logothetis CJ, Molina A, et al. Abiraterone and increased survival in metastatic prostate cancer. $N \mathrm{Engl} \mathrm{J} \mathrm{Med.}$ 2011;364(21):1995-2005.

6. Conn PM, Crowley WF Jr. Gonadotropin-releasing hormone and its analogues. N Engl J Med. 1991;324:93-103.

7. Lepor H. Comparison of single-agent androgen suppression for advanced prostate cancer. Rev Urol. 2005;7(suppl 1):S3-S12.

8. Waxman J, Man A, Hendry WF, et al. Importance of early tumour exacerbation in patients treated with long acting analogues of gonadotrophin releasing hormone for advanced prostatic cancer. Br Med J. 1985;291:1387-1388.

9. Thompson IM, Zeidman EJ, Rodriguez FR. Sudden death due to disease flare with luteinizing hormone-releasing hormone agonist therapy for carcinoma of the prostate. J Urol. 1990;144(6):1479-1480.

10. Klotz L, Boccon-Gibod L, Shore ND, et al. The efficacy and safety of degarelix: a 12-month, comparative, randomized, open-label, parallel-group phase III study in patients with prostate cancer. BJU Int. 2008;102(11):1531-1538.

11. Ezaki T, Kosaka T, Mizuno R, et al. Efficacy of treatment with a GnRH antagonist in prostate cancer patients previously treated with a $\mathrm{GnRH}$ agonist. Cancer Chemother Pharmacol. 2015;76(2):301-306.

12. Tombal B, Miller K, Boccon-Gibod L, et al. Additional analysis of the secondary end point of biochemical recurrence rate in a phase 3 trial (CS21) comparing degarelix $80 \mathrm{mg}$ versus leuprolide in prostate cancer patients segmented by baseline characteristics. Eur Urol. 2010;57(5):836-842.

13. Zattoni F. Prostate cancer: what are the news in hormonal therapy? The role of GnRH antagonists. Arch Ital Urol Androl. 2012;84(3):111-116.

14. Boccon-Gibod L, van der Meulen E, Persson BE. An update on the use of gonadotropin-releasing hormone antagonists in prostate cancer. Ther Adv Urol. 2011;3(3):127-140.

15. Sato T, Matsumoto T, Yamada T, Watanabe T, Kawano H, Kato S. Late onset of obesity in male androgen receptor-deficient (AR KO) mice. Biochem Biophys Res Commun. 2003;300(1):167-171.
16. Matsumoto T, Sakari M, Okada M, et al. The androgen receptor in health and disease. Annu Rev Physiol. 2013;75:201-224.

17. Keating NL, O'Malley AJ, Freedland SJ, Smith MR. Does comorbidity influence the risk of myocardial infarction or diabetes during androgendeprivation therapy for prostate cancer? Eur Urol. 2013;64(1):159-166.

18. Crawford ED, Shore ND, Moul JW, et al. Long-term tolerability and efficacy of degarelix: 5-year results from a phase III extension trial with a 1-arm crossover from leuprolide to degarelix. Urology. 2014;83(5):1122-1128.

19. Klotz L, Miller K, Crawford ED, et al. Disease control outcomes from analysis of pooled individual patient data from five comparative randomised clinical trials of degarelix versus luteinising hormone-releasing hormone agonists. Eur Urol. 2014;66(6):1101-1108.

20. Albertsen PC, Klotz L, Tombal B, Grady J, Olesen TK, Nilsson J. Cardiovascular morbidity associated with gonadotropin releasing hormone agonists and an antagonist. Eur Urol. 2014;65(3):565-573.

21. Collier A, Matthews DM, Kellett HA, Clarke BF, Hunter JA. Change in skin thickness associated with cheiroarthropathy in insulin dependent diabetes mellitus. Br Med J. 1986;292:936.

22. Richardson T, Kerr D. Skin-related complications of insulin therapy: epidemiology and emerging management strategies. Am J Clin Dermatol. 2003;4(10):661-667.

23. Crowther MA, Warkentin TE. Bleeding risk and the management of bleeding complications in patients undergoing anticoagulant therapy: focus on new anticoagulant agents. Blood. 2008;111(10):4871-4879.

24. Eikelboom JW, Wallentin L, Connolly SJ, et al. Risk of bleeding with 2 doses of dabigatran compared with warfarin in older and younger patients with atrial fibrillation: an analysis of the randomized evaluation of long-term anticoagulant therapy (RE-LY) trial. Circulation. 2011;123(21):2363-2372.

25. D'Amico AV, Whittington R, Malkowicz SB, et al. Biochemical outcome after radical prostatectomy, external beam radiation therapy, or interstitial radiation therapy for clinically localized prostate cancer. JAMA. 1998;280(11):969-974.

26. Tajima M, Noda M, Origasa H, et al. Evidence-based practice guideline for the treatment for diabetes in Japan 2013. Diabetol Int. 2015;6:151-187.

27. Ortel TL. Perioperative management of patients on chronic antithrombotic therapy. Blood. 2012;120(24):4699-4705.

28. Nomura S, Gilmour S, Tsubokura M, et al. Mortality risk amongst nursing home residents evacuated after the Fukushima nuclear accident: a retrospective cohort study. PLoS One. 2013;8(3):e60192.

29. Nomura S, Blangiardo M, Tsubokura M, et al. Post-nuclear disaster evacuation and survival amongst elderly people in Fukushima: a comparative analysis between evacuees and non-evacuees. Prev Med. 2016;82:77-82.

30. Miller LR, Partin AW, Chan DW, et al. Influence of radical prostatectomy on serum hormone levels. J Urol. 1998;160(2):449-453.

31. Olsson M, Ekström L, Schulze J, et al. Radical prostatectomy: influence on serum and urinary androgen levels. Prostate. 2010;70(2):200-205.

32. Lackner JE, Maerk I, Koller A, et al. Serum inhibin - not a cause of low testosterone levels in hypogonadal prostate cancer? Urology. 2008;72:1121-1124

33. Karunasinghe N, Masters J, Flanagan JU, Ferguson LR. Influence of Aldo-keto reductase $1 \mathrm{C} 3$ in prostate cancer-a mini review. Curr Cancer Drug Targets. 2017;17(7):603-616.

34. Karunasinghe N, Zhu Y, Han DY, et al. Quality of life effects of androgen deprivation therapy in a prostate cancer cohort in New Zealand: can we minimize effects using a stratification based on the aldo-keto reductase family 1 , member $\mathrm{C} 3 \mathrm{rs} 12529$ gene polymorphism? BMC Urol. 2016;16(1):48. 
Research and Reports in Urology is an international, peer-reviewed, open access journal publishing original research, reports, editorials, reviews and commentaries on all aspects of adult and pediatric urology in the clinic and laboratory including the following topics: Pathology, pathophysiology of urological disease; Investigation and treatment of urological disease; Pharmacology of drugs used for the treatment of urological disease. The manuscript management system is completely online and includes a very quick and fair peer-review system, which is all easy to use. Visit http://www.dovepress.com/testimonials.php to read real quotes from published authors

Submit your manuscript here: https://www.dovepress.com/research-and-reports-in-urology-journal 